\title{
Bifurcation Behaviors of Steady-State Solution to a Discrete General Brusselator Model
}

\author{
Ruyun Ma (D) and Zhongzi Zhao \\ Department of Mathematics, Northwest Normal University, Lanzhou 730070, China \\ Correspondence should be addressed to Ruyun Ma; mary@nwnu.edu.cn
}

Received 2 March 2020; Accepted 23 April 2020; Published 11 May 2020

Academic Editor: Giancarlo Consolo

Copyright (C) 2020 Ruyun Ma and Zhongzi Zhao. This is an open access article distributed under the Creative Commons Attribution License, which permits unrestricted use, distribution, and reproduction in any medium, provided the original work is properly cited.

We study the local and global bifurcation of nonnegative nonconstant solutions of a discrete general Brusselator model. We generalize the linear $u$ in the standard Brusselator model to the nonlinear $f(u)$. Assume that $f \in C([0, \infty),[0, \infty))$ is a strictly increasing function, and $f^{\prime}\left(f^{-1}(a)\right) \in(0, \infty)$. Taking $b$ as the bifurcation parameter, we obtain that the solution set of the problem constitutes a constant solution curve and a nonconstant solution curve in a small neighborhood of the bifurcation point $\left(b_{0}^{j}, f^{-1}(a), a b /\left[f^{-1}(a)\right]^{2}\right)$. Moreover, via the Rabinowitz bifurcation theorem, we obtain the global structure of the set of nonconstant solutions under the condition that $f(s) / s^{2}$ is nonincreasing in $(0, \infty)$. In this process, we also make a priori estimation for the nonnegative nonconstant solutions of the problem.

\section{Introduction}

In 1968, Prigogine and Lefever [1] introduced first the Brusselator model for a chemical reaction-diffusion of selfcatalysis as follows:

$$
\begin{cases}\frac{\partial u}{\partial t}-d_{1} \Delta u=a-(b+1) u+u^{2} v, & x \in \Omega, t>0, \\ \frac{\partial v}{\partial t}-d_{2} \Delta v=b u-u^{2} v, & x \in \Omega, t>0, \\ \frac{\partial u}{\partial n}=\frac{\partial v}{\partial n}=0, & x \in \partial \Omega, t>0,\end{cases}
$$

where $\Omega \subset \mathbb{R}^{N}(N \geq 1)$ is a smooth and bounded domain, $n$ denotes the outward unit normal vector on $\partial \Omega, u$ and $v$ represent the concentration of two intermediary reactants having the diffusion rates $d_{1}, d_{2} \in(0, \infty)$ with $d_{2}>d_{1}$, and $a, b>0$ are the fixed concentrations. This chemical reaction plays an important role due to its similarities with neuronal and biological networks. Therefore, (1) has been extensively investigated in the last decades from both analytical and numerical point of view (see [2-12]). Most of them are interested in finding spatially nonconstant solutions of the equilibrium problem

$$
\begin{cases}-d_{1} \Delta u=a-(b+1) u+u^{2} v, & x \in \Omega, \\ -d_{2} \Delta v=b u-u^{2} v, & x \in \Omega, \\ \frac{\partial u}{\partial n}=\frac{\partial v}{\partial n}=0, & x \in \partial \Omega .\end{cases}
$$

From the definition of Strogatz [13], chaos sensitivity depends on initial conditions, which shows that nearby trajectories diverge exponentially. Continuous systems in a 2-dimensional phase space cannot experience such divergence; hence, chaotic behaviors can only be observed in deterministic continuous systems with a phase space of dimension 3, at least. On the contrary, in a discrete map, it is well known that chaos occurs also in one dimension. Therefore, discrete chaotic systems exhibit chaos whatever their dimension is.

It is worth to note that discrete models governed by difference equations are more appropriate than the 
continuous one due to their efficient computational results and rich dynamical behavior (see $[14,15])$. Therefore, the discrete Brusselator model has been studied by several authors, and they got some results ([16-18] and the references therein). In particular, Din [16] applied forward Euler's method to one-dimensional model (1) as follows:

$$
\left\{\begin{array}{l}
x_{n+1}=x_{n}+h\left(\alpha-(1+\beta) x_{n}+x_{n}^{2} y_{n}\right), \\
y_{n+1}=y_{n}+h\left(\beta x_{n}-x_{n}^{2} y_{n}\right),
\end{array}\right.
$$

where $0<h<1$ represents the step size for Euler's method. The local dynamical behaviors are obtained for (3).

Note that [16-18] only studied the dynamical behaviors of the discrete-time Brusselator model. The reason is that the partial difference equation is very difficult for us. Indeed, the discrete-space Brusselator model is also worth studying due to the discontinuity of the space.

Therefore, we will consider the discrete space, more general form of (2) with $N=1$ :

$$
\begin{cases}-d_{1} \Delta^{2} u(x-1)=a-(b+1) f(u(x))+u^{2}(x) v(x), & x \in \mathbb{T}, \\ -d_{2} \Delta^{2} v(x-1)=b f(u(x))-u^{2}(x) v(x), & x \in \mathbb{T}, \\ \Delta u(0)=\Delta u(T)=\Delta v(0)=\Delta v(T)=0, & \end{cases}
$$

where $\Delta u(x)=u(x+1)-u(x), \mathbb{T}:=[1, T]_{\mathbb{Z}}=\{1,2, \ldots, T\}$, $T \geq 2$ is an integer, $d_{1}, d_{2}, a>0$ are fixed parameters and $d_{2}>d_{1}$, and $b>0$ is a bifurcation parameter. Clearly, $f(u)=(f(u) / u) \cdot u$; then, $(4)$ is seen to be equivalent to

$$
\begin{cases}-d_{1} \Delta^{2} u(x-1)=a-(b+1) \frac{f(u(x))}{u(x)} \cdot u(x)+u^{2}(x) v(x), & x \in \mathbb{T}, \\ -d_{2} \Delta^{2} v(x-1)=b \frac{f(u(x))}{u(x)} \cdot u(x)-u^{2}(x) v(x), & x \in \mathbb{T}, \\ \Delta u(0)=\Delta u(T)=\Delta v(0)=\Delta v(T)=0, & \end{cases}
$$

where $f(u) / u$ can be regarded as a variable coefficient. It is well known that the linear terms $(b+1) u$ and $b u$ in (2) cannot withstand any small perturbation.

In fact, (5) has an important application value in biology and chemistry. Xu et al. [19] said that model (1) includes a basic assumption: the cells always live in a continuous patch environment. However, this may not be the case in reality, and the motion of individuals of given cells is random and isotropic, i.e., without any preferred direction, the cells are also absolute individuals. The cells or units are also absolute individuals in microscopic sense, and each isolated cell exchanges materials by diffusion with its neighbors. Thus, it is reasonable to consider a $1 \mathrm{D}$ or $2 \mathrm{D}$ spatially discrete reaction-diffusion system in order to explain the chemical system.

Kang [20] discussed the dynamics of the local map of a discrete version of the Brusselator model. To discretize system (1), he employed the following discretizations.

For the derivative in time, he used

$$
\frac{\partial u(x, t)}{\partial t} \longrightarrow \frac{u(x, t+\Delta t)-u(x, t)}{\Delta t}
$$

For the space derivative, he used

$$
\begin{aligned}
& \frac{\partial u(x, t)}{\partial x} \longrightarrow \frac{u(x+\Delta x, t)-u(x, t)}{\Delta x}, \\
& \frac{\partial^{2} u(x, t)}{\partial x^{2}} \longrightarrow \frac{u(x+\Delta x, t)-2 u(x, t)+u(x-\Delta x, t)}{(\Delta x)^{2}} .
\end{aligned}
$$

It is important to note that $\Delta$ in (6)-(8) is different from $\Delta$ in this paper. Our discretization is consistent with Kang's, and we chose the step size to be 1 . When $f(u)=u,(5)$ is the steady-state form of the problem studied in $[19,20]$.

On the contrary, the Brusselator system has been investigated from the numerical point of view (see [21] and references therein). Most modern texts on numerical analysis give an introduction to numerical solutions of partial differential equations using the finite-difference approach. Twizell et al. [22] had given a second-order finitedifference scheme for the Brusselator reaction-diffusion system. It is well known that (2) is an important mathematical dynamics model in biology and chemistry. In some ways, (5) is even more practical than (2). 
We will study the local and global bifurcation of nonnegative nonconstant solutions of (4) under the following assumptions:

(H1) $f \in C([0, \infty),[0, \infty))$ is a strictly increasing function

(H2) $f^{\prime}\left(f^{-1}(a)\right) \in(0, \infty)$

(H3) $f(s) / s^{2}$ is nonincreasing in $(0, \infty)$

Remark 1. If $f(u)=u$, then (4) is the discrete version of (2) with $N=1$. Obviously, discrete Brusselator model (4) is a second-order difference boundary value problem.

The rest of the paper is organized as follows: in Section 2, we give a priori estimate and some preliminary results. Section 3 is devoted to studying the local bifurcation of nonnegative nonconstant solutions of (4) under conditions (H1) and (H2). Finally, in Section 4, we add condition (H3) to obtain the global bifurcation of nonnegative nonconstant solutions of (4).

\section{Preliminary Results}

At first, let us look for the constant solution of (4). To get it, it suffices to look for the constant solution of the following problem:

$$
\begin{cases}a-(b+1) f(u(x))+u^{2}(x) v(x)=0, & x \in \mathbb{T}, \\ b f(u(x))-u^{2}(x) v(x)=0, & x \in \mathbb{T} .\end{cases}
$$

By (H1), problem (4) has a unique constant solution $\left(f^{-1}(a), a b /\left[f^{-1}(a)\right]^{2}\right)$.

We can easily obtain the following a priori estimate of the nonnegative nonconstant solutions of (4).

Lemma 1. Let (H1), (H2), and (H3) hold. Then, any nonnegative nonconstant solution $(u, v)$ of $(4)$ satisfies

$$
\begin{array}{r}
f^{-1}\left(\frac{a}{b+1}\right) \leq u(x) \leq f^{-1}(a)+\frac{d_{2}}{d_{1}} \cdot \frac{a b}{(b+1)\left[f^{-1}(a /(b+1))\right]^{2}}, \quad x \in \mathbb{T}, \\
\frac{b f\left(f^{-1}(a)+\left(d_{2} / d_{1}\right) \cdot\left(a b /(b+1)\left[f^{-1}(a /(b+1))\right]^{2}\right)\right)}{\left[f^{-1}(a)+\left(d_{2} / d_{1}\right) \cdot\left(a b /(b+1)\left[f^{-1}(a /(b+1))\right]^{2}\right)\right]^{2}} \leq v(x) \leq \frac{a b}{(b+1)\left[f^{-1}(a /(b+1))\right]^{2}}, \quad x \in \mathbb{T} .
\end{array}
$$

Proof. Let $x_{0} \in \mathbb{T}$ be the minimum point of $u$. We have

$$
\begin{aligned}
u\left(x_{0}+1\right) & \geq u\left(x_{0}\right), \\
u\left(x_{0}-1\right) \geq & u\left(x_{0}\right), \\
-d_{1} \Delta^{2} u\left(x_{0}-1\right)= & -d_{1}\left(u\left(x_{0}+1\right)-2 u\left(x_{0}\right)\right. \\
& \left.+u\left(x_{0}-1\right)\right) \leq 0 .
\end{aligned}
$$

Then,

$$
\begin{aligned}
a-(b+1) f\left(u\left(x_{0}\right)\right)+u^{2}\left(x_{0}\right) v\left(x_{0}\right) & \leq 0, \\
a-(b+1) f\left(u\left(x_{0}\right)\right) & \leq 0, \\
f\left(u\left(x_{0}\right)\right) & \geq \frac{a}{b+1} .
\end{aligned}
$$

Then, $u\left(x_{0}\right) \geq f^{-1}(a /(b+1))$ by (H1), and so

$$
u(x) \geq u\left(x_{0}\right) \geq f^{-1}\left(\frac{a}{b+1}\right), \quad x \in \mathbb{T} .
$$

Let $x_{1} \in \mathbb{T}$ be the maximum point of $v$. Similarly, we can get that

$$
\begin{aligned}
v\left(x_{1}+1\right) & \leq v\left(x_{1}\right), v\left(x_{1}-1\right) \leq v(x), \\
-d_{2} \Delta v\left(x_{1}-1\right) & =-d_{1}\left(v\left(x_{1}+1\right)-2 v\left(x_{1}\right)+v\left(x_{1}-1\right)\right) \geq 0,
\end{aligned}
$$

$$
b f\left(u\left(x_{1}\right)\right)-u^{2}\left(x_{1}\right) v\left(x_{1}\right) \geq 0 .
$$

Then,

$$
v(x) \leq v\left(x_{1}\right) \leq \frac{b f\left(u\left(x_{1}\right)\right)}{u^{2}\left(x_{1}\right)}, \quad x \in \mathbb{T} .
$$

Combining this with (13), from (H3), we show

$$
v(x) \leq \frac{b f\left(u\left(x_{1}\right)\right)}{u^{2}\left(x_{1}\right)} \leq \frac{a b}{(b+1)\left[f^{-1}(a /(b+1))\right]^{2}}, \quad x \in \mathbb{T} \text {. }
$$

Let $w=d_{1} u+d_{2} v$. Then, it follows from (4) that

$$
\left\{\begin{array}{l}
-\Delta^{2} w(x-1)=a-f(u(x)), \quad x \in \mathbb{T}, \\
\Delta w(0)=\Delta w(T)=0 .
\end{array}\right.
$$
that

Now, let $x_{2} \in \mathbb{T}$ be the maximum point of $w$. Observe

$$
\begin{aligned}
w\left(x_{2}+1\right) & \geq w\left(x_{2}\right), \\
w\left(x_{2}-1\right) & \geq w\left(x_{2}\right), \\
-\Delta^{2} w\left(x_{2}-1\right) & =a-f\left(u\left(x_{2}\right)\right) \geq 0 .
\end{aligned}
$$

Then, from (H1), it is easy to see $u\left(x_{2}\right) \leq f^{-1}(a)$. Combining this with (17), we know that, for any $x \in \mathbb{T}$,

$$
\begin{aligned}
d_{1} u(x) \leq w(x) \leq w\left(x_{2}\right) \leq & d_{1} f^{-1}(a)+d_{2} \\
& \cdot \frac{a b}{(b+1)\left[f^{-1}(a /(b+1))\right]^{2}} .
\end{aligned}
$$


Then,

$u(x) \leq f^{-1}(a)+\frac{d_{2}}{d_{1}} \cdot \frac{a b}{(b+1)\left[f^{-1}(a /(b+1))\right]^{2}}, \quad x \in \mathbb{T}$.
If $x_{3} \in \mathbb{T}$ is the minimum point of $v$, then

$$
b f\left(u\left(x_{3}\right)\right)-u^{2}\left(x_{3}\right) v\left(x_{3}\right) \leq 0,
$$

and so

$$
v(x) \geq v\left(x_{3}\right) \geq \frac{b f\left(u\left(x_{3}\right)\right)}{u^{2}\left(x_{3}\right)} \geq \frac{b f\left(f^{-1}(a)+\left(d_{2} / d_{1}\right) \cdot a b /(b+1)\left[f^{-1}(a /(b+1))\right]^{2}\right)}{\left[f^{-1}(a)+\left(d_{2} / d_{1}\right) \cdot a b /(b+1)\left[f^{-1}(a /(b+1))\right]^{2}\right]^{2}}, \quad x \in \mathbb{T} .
$$

Consequently, the proof is completed.

Lemma 2 (see [23]). Assume $T \geq 2$ is an integer. Then, the discrete second-order linear Neumann eigenvalue problem

$$
\left\{\begin{array}{l}
-\Delta^{2} \varphi(x-1)=\mu \varphi(x), \quad x \in \mathbb{T}, \\
\Delta \varphi(0)=\Delta \varphi(T)=0
\end{array}\right.
$$

has $T$ real and simple eigenvalues, which can be ordered as follows:

$$
0=\mu_{0}<\mu_{1}<\mu_{2}<\cdots<\mu_{T-1} .
$$

Moreover, for $j \in\{1, \ldots, T-1\}$, the eigenfunction $\varphi_{j}$ corresponding to the eigenvalue $\mu_{j}$ has exactly $j-1$ simple generalized zeros.

For any fixed $T \geq 2$, it is well known that

$$
\mu_{j}= \begin{cases}0, & j=0, \\ 2-\cos \left(\frac{j \pi}{T}\right), & j \in(1, T-1]_{\mathbb{Z}},\end{cases}
$$

and the corresponding eigenfunctions are

$$
\varphi_{j}(x)= \begin{cases}1, & j=0, \\ \cos \left(\frac{j \pi}{T} x\right), & j \in(1, T-1]_{\mathbb{Z}} .\end{cases}
$$

Lemma 3 (see [18], Theorem 2.5). Let a be a constant. Then, for $\Delta C(i)=0$,

$$
\sum \cos a i=\frac{\sin a(i-(1 / 2))}{2 \sin (a / 2)}+C(i), \quad(a \neq 2 n \pi) .
$$

Lemma 4 (see [18], Theorem 2.7). If $z_{n}$ is an indefinite sum of $y_{n}$, then

$$
\sum_{k=m}^{n-1} y_{k}=\left[z_{k}\right]_{m}^{n}=z_{n}-z_{m}
$$

Let

$$
\begin{aligned}
X & :=\{(u, v) \mid u, v: \widehat{\mathbb{T}} \longrightarrow \mathbb{R}, \Delta u(0)=\Delta u(T)=\Delta v(0)=\Delta v(T)=0\}, \\
X_{1} & :=\{u \mid u: \mathbb{T} \longrightarrow \mathbb{R}\}, \quad Y:=X_{1} \times X_{1}, \\
\left(w_{1}, w_{2}\right)_{Y} & =\sum_{x=1}^{T} u_{1}(x) u_{2}(x)+\sum_{x=1}^{T} v_{1}(x) v_{2}(x),
\end{aligned}
$$

where $w_{1}=\left(u_{1}, v_{1}\right), w_{2}=\left(u_{2}, v_{2}\right) \in Y$ and $\widehat{\mathbb{T}}:=[0, T+1]_{\mathbb{Z}}$.

\section{Local Bifurcation}

By the second part, $\bar{w}:=\left(f^{-1}(a), a b /\left[f^{-1}(a)\right]^{2}\right)$ is the unique constant solution of (4).

Define the mapping $P:(0, \infty) \times X \longrightarrow Y$ :

$$
P(b, w)=\left(\begin{array}{c}
d_{1} \Delta^{2} u(x-1)+a-(b+1) f(u(x))+u^{2}(x) v(x) \\
d_{2} \Delta^{2} v(x-1)+b f(u(x))-u^{2}(x) v(x)
\end{array}\right) .
$$

For the fixed $b>0, w=(u, v)$ is a solution of (4) if and only if $(b, w)$ is a zero-point of $P$. Note that $P(b, \bar{w})=\left(\begin{array}{l}0 \\ 0\end{array}\right)$ since $\bar{w}$ is the constant solution of (4).
Let

$$
\left\{\begin{array}{c}
u=f^{-1}(a)+\sum_{k=1}^{\infty} \varepsilon^{k} u_{k}, \\
v=\frac{a b}{\left[f^{-1}(a)\right]^{2}}+\sum_{k=1}^{\infty} \varepsilon^{k} v_{k}, \\
b=b_{0}+\sum_{k=1}^{\infty} \varepsilon^{k} b_{k} .
\end{array}\right.
$$

We also have to Taylor expand $f$ at the point $f^{-1}(a)$. The purpose of the rest of this section is to solve $b_{0}$ and prove that $\left(b_{0}, \bar{w}\right)$ is the bifurcation point of $P(b, w)=\left(\begin{array}{l}0 \\ 0\end{array}\right)$. 
First of all, we substitute (32) and (33) into (4) and let the higher-order term of $\varepsilon$ be equal to 0 . Then, we can get the problem

$$
\begin{cases}-d_{1} \Delta^{2} u_{1}(x-1)=\left(-b_{0} f^{\prime}\left(f^{-1}(a)\right)-f^{\prime}\left(f^{-1}(a)\right)+\frac{2 a b_{0}}{f^{-1}(a)}\right) u_{1}(x)+\left[f^{-1}(a)\right]^{2} v_{1}(x), & x \in \mathbb{T}, \\ -d_{2} \Delta^{2} v_{1}(x-1)=\left(b_{0} f^{\prime}\left(f^{-1}(a)\right)-\frac{2 a b_{0}}{f^{-1}(a)}\right) u_{1}(x)-\left[f^{-1}(a)\right]^{2} v_{1}(x), & x \in \mathbb{T}, \\ \Delta u_{1}(0)=\Delta u_{1}(T)=\Delta v_{1}(0)=\Delta v_{1}(T)=0 . & \end{cases}
$$

In (34), by using undetermined coefficient method, it follows that

$$
b_{0}=\frac{f^{-1}(a)\left\{d_{1} d_{2} \mu_{j}^{2}+d_{2} f^{\prime}\left(f^{-1}(a)\right) \mu_{j}+d_{1}\left[f^{-1}(a)\right]^{2} \mu_{j}+\left[f^{-1}(a)\right]^{2} f^{\prime}\left(f^{-1}(a)\right)\right\}}{2 a d_{2} \mu_{j}-d_{2} f^{-1}(a) f^{\prime}\left(f^{-1}(a)\right) \mu_{j}}:=b_{0}^{j}, \quad j=1,2, \ldots T-1 .
$$

Moreover, it is not difficult to prove (34) has a nontrivial solution $\left(u_{1}, v_{1}\right)$ :

$$
\left\{\begin{array}{l}
u_{1}=c_{1}(j) \cos \left(\frac{j \pi}{l} x\right)=c_{1}(j) \varphi_{j}(x), \quad c_{1}(j)=-\frac{d_{2} \mu_{j}}{d_{1} \mu_{j}+f^{\prime}\left(f^{-1}(a)\right)} \\
v_{1}=\cos \left(\frac{j \pi}{l} x\right)=\varphi_{j}(x) .
\end{array}\right.
$$

Next, we substitute (32) and (33) into (4) and let the higher-order term of $\varepsilon^{2}$ be equal to 0 ; then, (4) becomes the following system:

$$
\begin{cases}d_{1} \Delta^{2} u_{2}(x-1)+\left(-b_{0} f^{\prime}\left(f^{-1}(a)\right)-f^{\prime}\left(f^{-1}(a)\right)+\frac{2 a b_{0}}{f^{-1}(a)}\right) u_{2}(x)+\left[f^{-1}(a)\right]^{2} v_{2}(x)=-F_{1}, & x \in \mathbb{T} \\ d_{2} \Delta^{2} v_{2}(x-1)+\left(b_{0} f^{\prime}\left(f^{-1}(a)\right)-\frac{2 a b_{0}}{f^{-1}(a)}\right) u_{2}(x)-\left[f^{-1}(a)\right]^{2} v_{2}(x)=F_{1}, & x \in \mathbb{T}, \\ \Delta u_{2}(0)=\Delta u_{2}(T)=\Delta v_{2}(0)=\Delta v_{2}(T)=0 & \end{cases}
$$

where

$$
F_{1}=\left(\frac{2 a b_{1}}{f^{-1}(a)}-b_{1} f^{\prime}\left(f^{-1}(a)\right)\right) u_{1}+2 f^{-1}(a) u_{1} v_{1}+\frac{a b_{0}}{\left[f^{-1}(a)\right]^{2}} u_{1}^{2} .
$$

In order to solve $b_{1}$ from (37), let us consider the following adjoint system of the homogeneous system related to (37): 


$$
\left\{\begin{array}{l}
d_{1} \Delta^{2} y_{2}(x-1)+\left(-b_{0} f^{\prime}\left(f^{-1}(a)\right)-f^{\prime}\left(f^{-1}(a)\right)+\frac{2 a b_{0}}{f^{-1}(a)}\right) y_{2}(x)+\left(b_{0} f^{\prime}\left(f^{-1}(a)\right)-\frac{2 a b_{0}}{f^{-1}(a)}\right) z_{2}(x)=0, \quad x \in \mathbb{T}, \\
d_{2} \Delta^{2} z_{2}(x-1)+\left[f^{-1}(a)\right]^{2} y_{2}(x)-\left[f^{-1}(a)\right]^{2} z_{2}(x)=0 \\
\Delta y_{2}(0)=\Delta y_{2}(T)=\Delta z_{2}(0)=\Delta z_{2}(T)=0 .
\end{array}\right.
$$

It is not difficult to verify that (39) has a solution $\left(y_{2}, z_{2}\right)$ :

$$
\left\{\begin{array}{l}
y_{2}=c_{2}(j) \cos \left(\frac{j \pi}{l} x\right)=c_{2}(j) \varphi_{j}(x), \quad c_{2}(j)=1+\frac{d_{2} \mu_{j}}{\left[f^{-1}(a)\right]^{2}}, \\
z_{2}=\cos \left(\frac{j \pi}{l} x\right)=\varphi_{j}(x) .
\end{array}\right.
$$

By virtue of the solvability condition for (37), it is obvious that

$$
\sum_{x=1}^{T}\left(z_{2}(x)-y_{2}(x)\right) F_{1}=0
$$

$$
\begin{aligned}
& \sum_{x=1}^{T}\left(z_{2}(x)-y_{2}(x)\right) F_{1} \\
& \quad=\sum_{x=1}^{T}\left(-\frac{d_{2} \mu_{j}}{\left[f^{-1}(a)\right]^{2}} \cdot \varphi_{j}(x)\right)\left[\left(\frac{2 a b_{1}}{f^{-1}(a)}-b_{1} f^{\prime}\left(f^{-1}(a)\right)\right) \cdot\left(-\frac{d_{2} \mu_{j}}{d_{1} \mu_{j}+f^{\prime}\left(f^{-1}(a)\right)} \cdot \varphi_{j}(x)\right)\right. \\
& \left.\quad+2 f^{-1}(a)\left(-\frac{d_{2} \mu_{j}}{d_{1} \mu_{j}+f^{\prime}\left(f^{-1}(a)\right)}\right) \varphi_{j}^{2}(x)+\frac{a b_{0}}{\left[f^{-1}(a)\right]^{2}} \cdot \frac{d_{2}^{2} \mu_{j}^{2}}{\left[d_{1} \mu_{j}+f^{\prime}\left(f^{-1}(a)\right)\right]^{2}} \cdot \varphi_{j}^{2}(x)\right]=0
\end{aligned}
$$

We know that

$\sum_{x=1}^{T} \varphi_{j}^{3}(x)=\sum_{x=1}^{T} \cos ^{3} \frac{j \pi}{T} x=\frac{1}{4} \sum_{x=1}^{T}\left(\cos \frac{3 j \pi}{T} x+3 \quad \cos \frac{j \pi}{T} x\right)$.
From Lemmas 3 and 4 , for any $j \in\{1,2, \ldots, T-1\}$ and $j \neq(2 n T / 3), n \in\{1,2, \ldots\}$, we obtain 


$$
\begin{aligned}
\sum_{x=1}^{T} \varphi_{j}^{3}(x) & =\frac{1}{4}\left[\frac{\sin (3 j \pi / T)(x-(1 / 2))}{2 \sin (3 j \pi / 2 T)}\right]_{1}^{T+1}+\frac{3}{4}\left[\frac{\sin (j \pi / T)(x-(1 / 2))}{2 \sin (j \pi / 2 T)}\right]_{1}^{T+1} \\
& =\frac{1}{4} \cdot \frac{\sin ((2 T+1) 3 j \pi / 2 T)-\sin (3 j \pi / 2 T)}{2 \sin (3 j \pi / 2 T)}+\frac{3}{4} \cdot \frac{\sin ((2 T+1) j \pi / 2 T)-\sin (j \pi / 2 T)}{2 \sin (j \pi / 2 T)} \\
& =\frac{1}{4} \cdot \frac{\cos ((2 T+2) 3 j \pi / 2 T) \cdot \sin (2 T \cdot 3 j \pi / 2 T)}{\sin (3 j \pi / 2 T)}+\frac{3}{4} \cdot \frac{\cos ((2 T+2) j \pi / 2 T) \cdot \sin (2 T \cdot j \pi / 2 T)}{\sin (j \pi / 2 T)}=0
\end{aligned}
$$

Then $b_{1}^{j}:=b_{1}=0$, and so $F_{1}$ will reduce to

$$
\begin{aligned}
F_{1} & =2 f^{-1}(a) c_{1}(j) \varphi_{j}^{2}(x)+\frac{a b_{0}}{\left[f^{-1}(a)\right]^{2}} c_{1}^{2}(j) \varphi_{j}^{2}(x) \\
& =2 f^{-1}(a) c_{1}(j) \cos ^{2}\left(\frac{j \pi}{l} x\right)+\frac{a b_{0}}{\left[f^{-1}(a)\right]^{2}} c_{1}^{2}(j) \cos ^{2}\left(\frac{j \pi}{l} x\right) \\
& =f^{-1}(a) c_{1}(j)\left(\cos \left(\frac{2 j \pi}{l} x\right)+1\right)+\frac{1}{2} \cdot \frac{a b_{0}}{\left[f^{-1}(a)\right]^{2}} c_{1}^{2}(j)\left(\cos \left(\frac{2 j \pi}{l} x\right)+1\right) \\
& =\frac{1}{2}\left[2 f^{-1}(a) c_{1}(j)+\frac{a b_{0}}{\left[f^{-1}(a)\right]^{2}} c_{1}^{2}(j)\right]+\frac{1}{2}\left[2 f^{-1}(a) c_{1}(j)+\frac{a b_{0}}{\left[f^{-1}(a)\right]^{2}} c_{1}^{2}(j)\right] \varphi_{2 j}(x) .
\end{aligned}
$$

Therefore, a particular solution $\left(u_{2}, v_{2}\right)$ of $(37)$ can be where obtained as follows:

$$
\left\{\begin{array}{l}
u_{2}=a_{1}(j)+a_{2}(j) \cos \left(\frac{2 j \pi}{l} x\right)=a_{1}(j)+a_{2}(j) \varphi_{2 j}(x), \\
v_{2}=a_{3}(j)+a_{4}(j) \cos \left(\frac{2 j \pi}{l} x\right)=a_{3}(j)+a_{4}(j) \varphi_{2 j}(x),
\end{array}\right.
$$

$$
\begin{aligned}
& a_{2}(j)=\frac{\left[f^{-1}(a)\right]^{2} c_{1} d_{2} \mu_{2 j}+\left(a b_{0} d_{2} \mu_{2 j} c_{1}^{2} / 2 f^{-1}(a)\right)}{d_{1} d_{2} \mu_{2 j}^{2} f^{-1}(a)+f^{\prime}\left(f^{-1}(a)\right) f^{-1}(a)\left(\left[f^{-1}(a)\right]^{2}+d_{2} \mu_{2 j}\left(1+b_{0}\right)\right)-\left(2 a b_{0} d_{2}-d_{1}\left[f^{-1}(a)\right]^{3}\right) \mu_{2 j}}, \\
& a_{1}(j)=0 \\
& a_{3}(j)=-\frac{c_{1}}{\left[f^{-1}(a)\right]^{2}}\left(f^{-1}(a)+\frac{a b_{0}}{2\left[f^{-1}(a)\right]^{2}} c_{1}\right), \\
& a_{4}(j)=-\frac{d_{1} \mu_{2 j}+f^{\prime}\left(f^{-1}(a)\right)}{d_{2} \mu_{2 j}} a_{2}(j) .
\end{aligned}
$$

Since $b_{1}=0$, we have to solve $b_{2}$. We substitute (32) and (33) into (4) and let the higher-order term of $\varepsilon^{3}$ be equal to 0 ; then, a problem similar to (37) is obtained: 


$$
\begin{cases}d_{1} \Delta^{2} u_{3}(x-1)+\left(-b_{0} f^{\prime}\left(f^{-1}(a)\right)-f^{\prime}\left(f^{-1}(a)\right)+\frac{2 a b_{0}}{f^{-1}(a)}\right) u_{3}(x)+\left[f^{-1}(a)\right]^{2} v_{3}(x)=-F_{2}, & x \in \mathbb{T}, \\ d_{2} \Delta^{2} v_{3}(x-1)+\left(b_{0} f^{\prime}\left(f^{-1}(a)\right)-\frac{2 a b_{0}}{f^{-1}(a)}\right) u_{3}(x)-\left[f^{-1}(a)\right]^{2} v_{3}(x)=F_{2}, & x \in \mathbb{T}, \\ \Delta u_{3}(0)=\Delta u_{3}(T)=\Delta v_{3}(0)=\Delta v_{3}(T) & \end{cases}
$$

where

$$
F_{2}=\left(-b_{2} f^{\prime}\left(f^{-1}(a)\right)+\frac{2 a b_{2}}{f^{-1}(a)}\right) u_{1}+2 f^{-1}(a) u_{1} v_{2}+2 f^{-1}(a) u_{2} v_{1}+u_{1}^{2} v_{1}+\frac{2 a b_{0}}{\left[f^{-1}(a)\right]^{2}} u_{1} u_{2} .
$$

Clearly, (39) is also the adjoint system of the homogeAccording to values of $u_{1}, u_{2}, v_{1}$, and $v_{2}$, we have neous system related to (48); then,

$$
\sum_{x=1}^{T}\left(z_{2}(x)-y_{2}(x)\right) F_{2}=0
$$

$$
\begin{aligned}
& \sum_{x=1}^{T}\left(z_{2}(x)-y_{2}(x)\right) F_{2} \\
& \quad=\sum_{x=1}^{T}\left(-\frac{d_{2} \mu_{j}}{\left[f^{-1}(a)\right]^{2}} \varphi_{j}(x)\right)\left[\frac{2 a-f^{\prime}\left(f^{-1}(a)\right) f^{-1}(a)}{f^{-1}(a)} \cdot b_{2} c_{1} \varphi_{j}(x)+2 f^{-1}(a) c_{1} \varphi_{j}(x)\left(a_{3}+a_{4}\right) \varphi_{2 j}(x)\right. \\
& \left.\quad+2 f^{-1}(a) a_{2} \varphi_{2 j}(x) \varphi_{j}(x)+c_{1}^{2} \varphi_{j}^{3}(x)+\frac{2 a b_{0} a_{2} c_{1}}{\left[f^{-1}(a)\right]^{2}} \varphi_{j}(x) \varphi_{2 j}(x)\right] .
\end{aligned}
$$

From Lemmas 3 and 4 , for any $j \in\{1,2, \ldots, T-1\}$ and $j \neq(n T / 2), n \in\{1,2, \ldots\}$, we know that

$$
\begin{aligned}
& \sum_{x=1}^{T} \varphi_{j}^{2}(x) \varphi_{2 j}(x)=\sum_{x=1}^{T} \cos ^{2}\left(\frac{j \pi}{T} x\right) \cdot \cos \left(\frac{2 j \pi}{T} x\right)=\frac{1}{2} \sum_{x=1}^{T}\left(\frac{1}{2} \cos \frac{4 j \pi}{T} x+\cos \frac{2 j \pi}{T} x\right)+\frac{T}{4} \\
&=\frac{1}{4} \cdot\left[\frac{\sin (4 j \pi(x-(1 / 2)) / T)}{2 \sin (4 j \pi / 2 T)}\right]_{1}^{T+1}+\frac{1}{2} \cdot\left[\frac{\sin (2 j \pi(x-(1 / 2)) / T)}{2}\right]_{1}^{T+1}+\frac{T}{4} \\
&\left.=\frac{1}{4} \cdot \frac{2 \cos ((2 j \pi / 2 T)}{2 \sin (2 j \pi / T)} 2 j \pi / T\right) \sin ((2 j \pi \cdot 2 T) / T) \\
& \frac{1}{2} \cdot \frac{2 \cos ((2 T+2) j \pi / T) \sin ((j \pi \cdot 2 T) / T)}{2 \sin (2 j \pi / T)}+\frac{T}{4}=\frac{T}{4} \neq 0
\end{aligned}
$$

Thus,

$$
b_{2}^{j}:=b_{2} \neq 0, \quad j=1,2, \ldots T-1 .
$$

From the above analysis, we obtain the main result of this section.

Theorem 1. Assume that (H1) and (H2) hold. Then, for any positive integer $j<T, j \neq(n T / 2)$ and $j \neq(2 n T / 3), n \in\{1,2$, $\ldots\}, \quad\left(b_{0}^{j}, \bar{w}\right)$ is a bifurcation point of $P(b, w)=\left(\begin{array}{l}0 \\ 0\end{array}\right)$. Moreover, there is a nontrivial solution $\phi(\varepsilon)=(b(\varepsilon)$, 
$u(\varepsilon), v(\varepsilon))$ of (4) if $\varepsilon$ is small enough, where $b, u$, and $v$ are continuous with respect to $\varepsilon$ :

$$
\begin{aligned}
& u(\varepsilon)=f^{-1}(a)+\varepsilon c_{1}(j) \varphi_{j}+\varepsilon^{2}\left(a_{1}(j)+a_{2}(j) \varphi_{2 j}\right)+o\left(\varepsilon^{2}\right), \\
& v(\varepsilon)=\frac{a b}{\left[f^{-1}(a)\right]^{2}}+\varepsilon \varphi_{j}+\varepsilon^{2}\left(a_{3}(j)+a_{4}(j) \varphi_{2 j}\right)+o\left(\varepsilon^{2}\right), \\
& b(\varepsilon)=b_{0}^{j}+\varepsilon^{2} b_{2}^{j}+o\left(\varepsilon^{2}\right) .
\end{aligned}
$$

The set of zero-points of $P$ constitutes two curves in a neighborhood of bifurcation point $\left(b_{0}^{j}, \bar{w}\right)$.

Let $\mathbb{C}$ be the closure of the nonconstant solution set of $P(b, w)=\left(\begin{array}{l}0 \\ 0\end{array}\right)$ and $\Gamma_{j}$ be a connected component of $\mathbb{C} \cup\left\{\left(b_{0}^{j}, \bar{w}\right)\right\}$ and $\left(b_{0}^{j}, \bar{w}\right) \in \Gamma_{j}$. In a small neighborhood of bifurcation point $\left(b_{0}^{j}, \bar{w}\right)$, the curve $\Gamma_{j}$ is determined by the eigenfunction $\varphi_{j}$, where $\varphi_{j}$ has exactly $j-1$ simple generalized zeros.

\section{Global Bifurcation}

Theorem 2. Let (H1), (H2), and (H3) hold. If $\mu_{j} \neq\left[f^{-1}(a)\right]^{2} / d_{2}, j=1,2, \ldots, T$, then projection of continuum $\Gamma_{j}$ is unbounded on the b-axis.

Proof. (4) can be written as follows:

$$
\begin{cases}-\Delta^{2} u(x-1)=g(u(x), v(x)), & x \in \mathbb{T}, \\ -\Delta^{2} v(x-1)=h(u(x), v(x)), & x \in \mathbb{T}, \\ \Delta u(0)=\Delta u(T)=\Delta v(0)=\Delta v(T)=0, & \end{cases}
$$

where

$$
\begin{aligned}
& g(u, v)=\frac{1}{d_{1}}\left(a-(b+1) f(u)+u^{2} v\right), \\
& h(u, v)=\frac{1}{d_{2}}\left(b f(u)-u^{2} v\right) .
\end{aligned}
$$

Let $\widetilde{u}=u-f^{-1}(a), \widetilde{v}=v-a b /\left[f^{-1}(a)\right]^{2}$. Then, (55) is equivalent to the following problem:

$$
\begin{cases}-\Delta^{2} \widetilde{u}(x-1)=g_{0} \widetilde{u}(x)+g_{1} \widetilde{v}(x)+\widetilde{g}(\widetilde{u}(x), \widetilde{v}(x)), & x \in \mathbb{T}, \\ -\Delta^{2} \widetilde{v}(x-1)=h_{0} \widetilde{u}(x)+h_{1} \widetilde{v}(x)+\widetilde{h}(\widetilde{u}(x), \widetilde{v}(x)), & x \in \mathbb{T}, \\ \Delta \widetilde{u}(0)=\Delta \widetilde{u}(T)=\Delta \widetilde{v}(0)=\Delta \widetilde{v}(T)=0, & \end{cases}
$$

where $\tilde{g}$ and $\widetilde{h}$ are higher-order terms of $\widetilde{u}, \widetilde{v}$ and

$$
\begin{array}{r}
g_{0}=\left.g_{u}(u, v)\right|_{\left(f^{-1}(a), a b /\left[f^{-1}(a)\right]^{2}\right)}=\frac{1}{d_{1}}\left(-(b+1) f^{\prime}\left(f^{-1}(a)\right)+\frac{2 a b}{f^{-1}(a)}\right) \\
g_{1}=\left.g_{v}(u, v)\right|_{\left(f^{-1}(a), a b /\left[f^{-1}(a)\right]^{2}\right)}=\frac{\left[f^{-1}(a)\right]^{2}}{d_{1}} \\
h_{0}=\left.h_{u}(u, v)\right|_{\left(f^{-1}(a), a b /\left[f^{-1}(a)\right]^{2}\right)}=\frac{1}{d_{2}}\left(b f^{\prime}\left(f^{-1}(a)\right)-\frac{2 a b}{f^{-1}(a)}\right) \\
h_{1}=\left.h_{v}(u, v)\right|_{\left(f^{-1}(a), a b /\left[f^{-1}(a)\right]^{2}\right)}=-\frac{\left[f^{-1}(a)\right]^{2}}{d_{2}}
\end{array}
$$

In this way, we convert the constant solution $\bar{w}=\left(f^{-1}(a), a b /\left[f^{-1}(a)\right]^{2}\right)$ of $(4)$ to the trivial solution $\theta=$ $(0,0)$ of $(57)$.

Let $H_{1}: Y \longrightarrow X$ and $H_{2}: Y \longrightarrow X$ be the inverse of operators $\left(f^{\prime}\left(f^{-1}(a)\right) / d_{1}\right) I-A$ and $\left(\left[f^{-1}(a)\right]^{2} / d_{2}\right) I-A$ with Neumann boundary conditions, respectively, where $A:[0, \infty) \longrightarrow(-\infty, 0]$ and $A u(x)=\Delta^{2} u(x-1)$. Set $U=(\widetilde{u}, \widetilde{v}):$

$$
\begin{aligned}
K(b) U & =\left(\frac{1}{d_{1}}\left[-b f^{\prime}\left(f^{-1}(a)\right)+\frac{2 a b}{f^{-1}(a)}\right] H_{1}(\widetilde{u})+\frac{\left[f^{-1}(a)\right]^{2}}{d_{1}} H_{1}(\widetilde{v}), \frac{1}{d_{2}}\left[b f^{\prime}\left(f^{-1}(a)\right)-\frac{2 a b}{f^{-1}(a)}\right] H_{2}(\widetilde{u})\right), \\
W(U) & =\left(H_{1}(\widetilde{g}(\widetilde{u}, \widetilde{v})), H_{2}(\widetilde{h}(\widetilde{u}, \widetilde{v}))\right) .
\end{aligned}
$$


It can be verified that (57) is equivalent to

$$
U=K(b) U+W(U),
$$

in $X$. For any fixed $b>0, K(b)$ and $W(U)$ are linear compact operators in $X$ and $W(U)=o(\|U\|)$. By the Rabinowitz global bifurcation theorem [24], we need to verify

(i) 1 is an eigenvalue of $K\left(b_{0}^{j}\right)$, and its algebraic multiplicity is 1 (ii) The index of $I-K(b)-W$ changes when $b$ crosses $b_{0}^{j}$ Now, we will prove (i). Suppose $\Psi=\left(\begin{array}{c}\xi \\ \psi\end{array}\right), \xi=\sum a_{j} \varphi_{j}$,
$\psi=\sum c_{j} \varphi_{j}$. Let

$$
(K(b)-I) \Psi=\left(\begin{array}{l}
0 \\
0
\end{array}\right)
$$

i.e.,

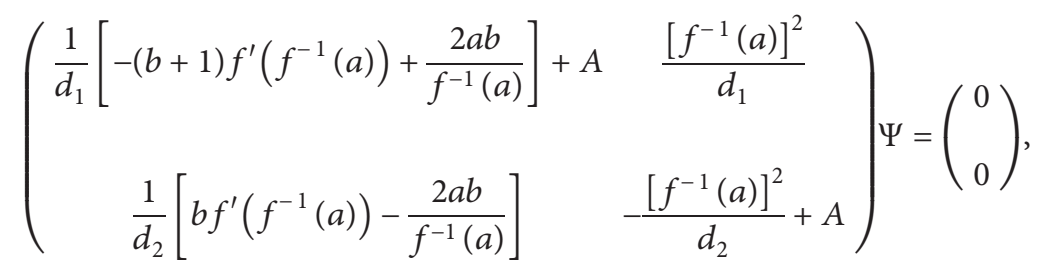

Thus,

where

$$
\sum_{j=0}^{\infty} L_{j}\left(\begin{array}{c}
a_{j} \\
c_{j}
\end{array}\right) \varphi_{j}=\left(\begin{array}{l}
0 \\
0
\end{array}\right)
$$

$$
L_{j}=\left(\begin{array}{cc}
\frac{1}{d_{1}}\left[-(b+1) f^{\prime}\left(f^{-1}(a)\right)+\frac{2 a b}{f^{-1}(a)}\right]-\mu_{j} & \frac{\left[f^{-1}(a)\right]^{2}}{d_{1}} \\
\frac{1}{d_{2}}\left[b f^{\prime}\left(f^{-1}(a)\right)-\frac{2 a b}{f^{-1}(a)}\right] & -\frac{\left[f^{-1}(a)\right]^{2}}{d_{2}}-\mu_{j}
\end{array}\right) .
$$

By computation, $\operatorname{det} L_{j}=0$ if and only if $b=b_{0}^{j}$; taking $b=b_{0}^{j}$ leads to

$$
L_{j}\left(\begin{array}{c}
a_{j} \\
c_{j}
\end{array}\right)=\left(\begin{array}{cc}
0 & 0 \\
f^{\prime}\left(f^{-1}(a)\right)+d_{1} \mu_{j} & d_{2} \mu_{j}
\end{array}\right)\left(\begin{array}{c}
a_{j} \\
c_{j}
\end{array}\right) .
$$

$$
\operatorname{ker}\left(K\left(b_{0}^{j}\right)-I\right)=\operatorname{span}(\Psi), \Psi=
$$

eigenvalue of $K=K\left(b_{0}^{j}\right)$ and dimker $(K-I)=1$. The algebraic multiplicity of eigenvalue 1 is the dimension of the generalized null space $\cup_{i=1}^{\infty} \operatorname{ker}(K-I)^{i}$; therefore, $\operatorname{ker}(K-I)$ $\cap \operatorname{Im}(K-I)=\left\{\theta^{T}\right\}$.

Let $K^{T}$ be the transposed matrix of $K$ : $\left(\begin{array}{l}\text { Then }{ }_{2} d_{2} \mu_{j} \\ f^{\prime}\left(f^{-1}(a)\right)+d_{1} \mu_{j}\end{array}\right) \varphi_{j} . \begin{aligned} & \operatorname{ker}\left(K\left(b_{0}^{j}\right)-I\right)=\operatorname{span}(\Psi), \Psi= \\ & \text { This implies that } 1 \text { is the }\end{aligned}$

$$
K^{T}=\left(\begin{array}{cc}
\frac{1}{d_{1}}\left[-b f^{\prime}\left(f^{-1}(a)\right)+\frac{2 a b}{f^{-1}(a)}\right] H_{1} & \frac{1}{d_{2}}\left[b f^{\prime}\left(f^{-1}(a)\right)-\frac{2 a b}{f^{-1}(a)}\right] H_{2} \\
\frac{\left[f^{-1}(a)\right]^{2}}{d_{1}} H_{1} & 0
\end{array}\right),
$$

and $\quad \Psi^{*}=\left(\begin{array}{c}\xi^{*} \\ \psi^{*}\end{array}\right), \xi^{*}=\sum a_{j}^{*} \varphi_{j}, \psi^{*}=\sum c_{j}^{*} \varphi_{j} . \quad$ Suppose $\Psi^{*} \in \operatorname{ker}\left(K^{T}-I\right)$. Then, 


$$
\left\{\begin{array}{l}
\frac{1}{d_{1}}\left[-b f^{\prime}\left(f^{-1}(a)\right)+\frac{2 a b}{f^{-1}(a)}\right] H_{1}\left(\xi^{*}\right)+\frac{1}{d_{2}}\left[b f^{\prime}\left(f^{-1}(a)\right)-\frac{2 a b}{f^{-1}(a)}\right] H_{2}\left(\psi^{*}\right)=\xi^{*}, \\
\frac{\left[f^{-1}(a)\right]^{2}}{d_{1}} H_{1}\left(\xi^{*}\right)=\psi^{*} .
\end{array}\right.
$$

From the definition of $H_{1}$ and $H_{2}$, (67) can also be written as

$$
\left\{\begin{array}{l}
-d_{1} d_{2} \Delta^{2} \xi^{*}(x-1)=\left(d_{2}\left[-b f^{\prime}\left(f^{-1}(a)\right)+\frac{2 a b}{f^{-1}(a)}\right]-\left[f^{-1}(a)\right]^{2} d_{1}\right) \xi^{*}-\frac{d_{2} f^{\prime}\left(f^{-1}(a)\right)}{\left[f^{-1}(a)\right]^{2}}\left[-b f^{\prime}\left(f^{-1}(a)\right)+\frac{2 a b}{f^{-1}(a)}\right] \psi^{*}, \\
-d_{1} \Delta^{2} \psi^{*}(x-1)=\left[f^{-1}(a)\right]^{2} \xi^{*}(x)-f^{\prime}\left(f^{-1}(a)\right) \psi^{*}(x) .
\end{array}\right.
$$

That is to say, where

$$
\sum_{j=0}^{\infty} B_{j}\left(\begin{array}{c}
a_{j}^{*} \\
c_{j}^{*}
\end{array}\right) \varphi_{j}=\left(\begin{array}{l}
0 \\
0
\end{array}\right),
$$

$$
B_{j}=\left(\begin{array}{cc}
d_{2}\left[-b f^{\prime}\left(f^{-1}(a)\right)+\frac{2 a b}{f^{-1}(a)}\right]-\left[f^{-1}(a)\right]^{2} d_{1}-d_{1} d_{2} \mu_{j} & -\frac{d_{2} f^{\prime}\left(f^{-1}(a)\right)}{\left[f^{-1}(a)\right]^{2}}\left[-b f^{\prime}\left(f^{-1}(a)\right)+\frac{2 a b}{f^{-1}(a)}\right] \\
{\left[f^{-1}(a)\right]^{2}} & -d_{1} \mu_{j}-f^{\prime}\left(f^{-1}(a)\right)
\end{array}\right) .
$$

Similarly, $\operatorname{det} B_{j}=0$ if and only if $b=b_{0}^{j}$; taking $b=b_{0}^{j}$ leads to

$$
B_{j}\left(\begin{array}{c}
a_{j}^{*} \\
c_{j}^{*}
\end{array}\right)=\left(\begin{array}{cc}
0 & 0 \\
{\left[f^{-1}(a)\right]^{2}} & -d_{1} \mu_{j}-f^{\prime}\left(f^{-1}(a)\right)
\end{array}\right)\left(\begin{array}{c}
a_{j}^{*} \\
c_{j}^{*}
\end{array}\right) .
$$

$$
\begin{aligned}
\left(\Psi, \Psi^{*}\right)_{Y} & =\sum_{x=1}^{T}\left(-d_{2} \mu_{j}\right)\left(d_{1} \mu_{j}+f^{\prime}\left(f^{-1}(a)\right)\right) \varphi_{j}(x)+\sum_{x=1}^{T}\left(f^{\prime}\left(f^{-1}(a)\right)+d_{1} \mu_{j}\right)\left[f^{-1}(a)\right]^{2} \varphi_{j}^{2}(x) \\
& =\left(d_{1} \mu_{j}+f^{\prime}\left(f^{-1}(a)\right)\right)\left(\left[f^{-1}(a)\right]^{2}-d_{2} \mu_{j}\right) \sum_{x=1}^{T} \varphi_{j}^{2}(x) \\
& =\left(d_{1} \mu_{j}+f^{\prime}\left(f^{-1}(a)\right)\right)\left(\left[f^{-1}(a)\right]^{2}-d_{2} \mu_{j}\right) \sum_{x=1}^{T} \cos ^{2}\left(\frac{j \pi}{T} x\right) \\
& =\left(d_{1} \mu_{j}+f^{\prime}\left(f^{-1}(a)\right)\right)\left(\left[f^{-1}(a)\right]^{2}-d_{2} \mu_{j}\right) \cdot \frac{1}{2} \sum_{x=1}^{T}\left(\cos \left(\frac{2 j \pi}{T} x\right)+1\right) \\
& =\left(d_{1} \mu_{j}+f^{\prime}\left(f^{-1}(a)\right)\right)\left(\left[f^{-1}(a)\right]^{2}-d_{2} \mu_{j}\right)\left[\frac{1}{2} \cdot \frac{\cos ((2 T+2) j \pi / T) \sin \quad 2 \quad \pi}{\sin (j \pi / T)}+\frac{T}{2}\right] \neq 0 .
\end{aligned}
$$


This suggests that $\Psi \notin\left(\operatorname{ker}\left(K^{*}-I\right)\right)^{\perp}=\operatorname{Im}(K-I)$, and so (i) is proved.

Now, we will prove (ii). From (i), for any $b>0, b \neq b_{0}^{j}$ and $b$ belongs to a small neighborhood of $b_{0}^{j}$, and $K(b)-I: X \longrightarrow X$ is a bijection. Fix $b>0$; then, $\theta$ is a solution of (60), and $\theta$ is isolated. From Leray-Schauder fixed point theory, we can get
$\operatorname{index}(I-K(b)-W,(b, \theta))=\operatorname{deg}(I-K(b), B, \theta)=(-1)^{\gamma}$,

where $B$ is a sufficiently small ball centered at $\theta, \gamma$ is the sum of the algebraic multiplicity of the eigenvalues of $K(b)$, and $\gamma>1$.

We are going to verify that, for $\varepsilon>0$ is small enough,

$$
\text { index }\left(I-K\left(b_{0}^{j}-\varepsilon\right)-W,\left(b_{0}^{j}-\varepsilon, \theta\right)\right) \neq \operatorname{index}\left(I-K\left(b_{0}^{j}+\varepsilon\right)-W,\left(b_{0}^{j}+\varepsilon, \theta\right)\right) \text {. }
$$
If $\tau$ is an eigenvalue of $K(b)$ and $\Psi=\left(\begin{array}{c}\xi \\ \psi\end{array}\right)$ is the i.e.,
corresponding eigenfunction, then

$$
(K(b)-I) \Psi=\left(\begin{array}{l}
0 \\
0
\end{array}\right),
$$

$$
\begin{cases}-\tau d_{1} \Delta^{2} \xi(x-1)=\left(-b f^{\prime}\left(f^{-1}(a)\right)+\frac{2 a b}{f^{-1}(a)}-\tau f^{\prime}\left(f^{-1}(a)\right)\right) \xi(x)+\left[f^{-1}(a)\right]^{2} \psi(x), & x \in \mathbb{T}, \\ -\tau d_{2} \Delta^{2} \psi(x-1)=\left(b f^{\prime}\left(f^{-1}(a)\right)-\frac{2 a b}{f^{-1}(a)}\right) \xi(x)-\left[f^{-1}(a)\right]^{2} \tau \psi(x), & x \in \mathbb{T} .\end{cases}
$$

By virtue of $\xi=\sum a_{j} \varphi_{j}$ and $\psi=\sum c_{j} \varphi_{j}$, we can get

$$
\sum_{j=0}^{\infty}\left(\begin{array}{cc}
\tau \mu_{j} d_{1}+b f^{\prime}\left(f^{-1}(a)\right)-\frac{2 a b}{f^{-1}(a)}+\tau f^{\prime}\left(f^{-1}(a)\right) & -\left[f^{-1}(a)\right]^{2} \\
-b f^{\prime}\left(f^{-1}(a)\right)+\frac{2 a b}{f^{-1}(a)} & \tau \mu_{j} d_{2}+\tau\left[f^{-1}(a)\right]^{2}
\end{array}\right)\left(\begin{array}{l}
a_{j} \\
c_{j}
\end{array}\right) \varphi_{j}=\left(\begin{array}{l}
0 \\
0
\end{array}\right) .
$$

Then, the characteristic equation is

$$
\begin{aligned}
& \left(d_{1} d_{2} \mu_{j}^{2}+d_{2} \mu_{i} f^{\prime}\left(f^{-1}(a)\right)+d_{1} \mu_{j}\left[f^{-1}(a)\right]^{2}+f^{\prime}\left(f^{-1}(a)\right)\left[f^{-1}(a)\right]^{2}\right) \tau^{2} \\
& +b\left(d_{2} f^{\prime}\left(f^{-1}(a)\right) \mu_{j}-\frac{2 a d_{2} \mu_{j}}{f^{-1}(a)}+f^{\prime}\left(f^{-1}(a)\right)\left[f^{-1}(a)\right]^{2}-2 a f^{-1}(a)\right) \tau+\left[f^{-1}(a)\right]^{2}\left(-b f^{\prime}\left(f^{-1}(a)\right)+\frac{2 a b}{f^{-1}(a)}\right)=0, \\
& j=0,1,2, \ldots, T .
\end{aligned}
$$


If $\tau=1, b$ can be solved from (78):

$$
b=\frac{f^{-1}(a)\left\{d_{1} d_{2} \mu_{j}^{2}+d_{2} f^{\prime}\left(f^{-1}(a)\right) \mu_{j}+d_{1}\left[f^{-1}(a)\right]^{2} \mu_{j}+\left[f^{-1}(a)\right]^{2} f^{\prime}\left(f^{-1}(a)\right)\right\}}{2 a d_{2} \mu_{j}-d_{2} f^{-1}(a) f^{\prime}\left(f^{-1}(a)\right) \mu_{j}}=b_{0}^{j} .
$$

Therefore, by calculating the corresponding eigenvalues of (78), we can obtain that when $b$ passes through $b_{0}^{j}$, the number of eigenvalues of $K(b)$ which is greater than 1 is the same, and their algebraic multiplicity are equal. By plugging (79) into (78), we have

$$
\begin{aligned}
& \frac{2 a d_{2} \mu_{j}-d_{2} f^{-1}(a) f^{\prime}\left(f^{-1}(a)\right) \mu_{j}}{f^{-1}(a)} \tau^{2}+\left(f^{\prime}\left(f^{-1}(a)\right)\left(d_{2} \mu_{j}+\left[f^{-1}(a)\right]^{2}\right)-\frac{2 a d_{2} \mu_{j}}{f^{-1}(a)}-2 a f^{-1}(a)\right) \tau \\
& +\left[f^{-1}(a)\right]^{2}\left(-f^{\prime}\left(f^{-1}(a)\right)+\frac{2 a}{f^{-1}(a)}\right)=0 .
\end{aligned}
$$

Then,

$$
\begin{gathered}
\left(d_{2} f^{\prime}\left(f^{-1}(a)\right) \mu_{j}-\frac{2 a d_{2} \mu_{j}}{f^{-1}(a)}+f^{\prime}\left(f^{-1}(a)\right)\left[f^{-1}(a)\right]^{2}-2 a f^{-1}(a)\right)^{2}-4\left(2 a d_{2} \mu_{j}-d_{2} f^{-1}(a) f^{\prime}\left(f^{-1}(a)\right) \mu_{j}\right) \\
\cdot\left(-f^{\prime}\left(f^{-1}(a)\right) f^{-1}(a)+2 a\right)=\left[\left(d_{2} f^{\prime}\left(f^{-1}(a)\right) \mu_{j}-\frac{2 a d_{2} \mu_{j}}{f^{-1}(a)}\right)-\left(f^{\prime}\left(f^{-1}(a)\right)\left[f^{-1}(a)\right]^{2}-2 a f^{-1}(a)\right)\right]^{2}>0,
\end{gathered}
$$

and so (80) has two different roots $\tau_{1}=1, \tau_{2}=\left[f^{-1}(a)\right]^{2} /$ $d_{2} \mu_{j}$. Thus, two things will happen:

(a) If $\mu_{j}>\left[f^{-1}(a)\right]^{2} / d_{2}$, then $\tau_{1}\left(b_{0}^{j}\right)=1, \tau_{2}\left(b_{0}^{j}\right)<1$.

(b) If $\mu_{j}<\left[f^{-1}(a)\right]^{2} / d_{2}$, then $\tau_{1}\left(b_{0}^{j}\right)=1, \tau_{2}\left(b_{0}^{j}\right)>1$.

When scenario (a) occurs, $b$ passes through $b_{0}^{j}$ and $\tau_{2}(b)<1$. From (78), $\tau_{1}\left(b_{0}^{j}+\varepsilon\right)>1, \tau_{1}\left(b_{0}^{j}-\varepsilon\right)<1$. Therefore, the matrix $K\left(b_{0}^{j}+\varepsilon\right)$ has exactly one more eigenvalue that is $>1$ than $K\left(b_{0}^{j}-\varepsilon\right)$ does, and its algebraic multiplicity is 1 . Then, (74) holds. That is to say, the index jumps as $b$ goes through $b_{0}^{j}$.

When scenario (b) occurs, $b$ passes through $b_{0}^{j}$ and $\tau_{2}(b)>1$. From (78), $\tau_{1}\left(b_{0}^{j}+\varepsilon\right)>1, \tau_{1}\left(b_{0}^{j}-\varepsilon\right)<1$. Similarly, the index jumps as $b$ goes through $b_{0}^{j}$. Therefore, (ii) is true regardless of (a) or (b).

Thus, by the index jump principle and [24], Theorem 1.3, it follows that there exists a connected component $\widehat{\Gamma}_{j}$ of nontrivial solutions of (60), and $\widehat{\Gamma}_{j}$ comes from the bifurcation point $\left(b_{0}^{j}, \theta\right)$. We know that $\widehat{\Gamma}_{j}$ is also the connected component $\Gamma_{j}$ of the nonconstant solution of (4) from $\left(b_{0}^{j}, \bar{w}\right) . \widehat{\Gamma}_{j}$ and $\Gamma_{j}$ are both in $\mathbb{R} \times X$. By the Rabinowitz global bifurcation theorem, the connected component $\Gamma_{j}$ joins $\left(b_{0}^{j}, \bar{w}\right)$ to either $\infty$ or $\left(b_{0}^{k}, \bar{w}\right)$ in $\mathbb{R} \times X$, where $k \neq j$.

We first prove that the latter situation will not happen. According to Theorem 1, the solution on the connected component sent from $\left(b_{0}^{j}, \bar{w}\right)$ is related to $\varphi_{j}$, and $\varphi_{j}$ has exactly $j-1$ simple generalized zeros. In the same way, the solution on the connected component sent from $\left(b_{0}^{k}, \bar{w}\right)$ is related to $\varphi_{k}$, and $\varphi_{k}$ has $k-1$ simple generalized zeros. If the connected component sent $\Gamma_{j}$ joining $\left(b_{0}^{j}, \bar{w}\right)$ to $\left(b_{0}^{k}, \bar{w}\right)$, the solution $(b, w) \in \Gamma_{j}$ is related to both $\varphi_{j}$ and $\varphi_{k}$, which is impossible. On the contrary, Lemma 1 shows that if $b=b_{c} \in(0, \infty)$, then the solutions $u$ and $v$ of (4) are both bounded. So, the connected component $\Gamma_{j}$ will not join $\left(b_{0}^{j}, \bar{w}\right)$ to $\left(b_{c}, \infty\right)$. Therefore, the connected component $\Gamma_{j}$ can only join $\left(b_{0}^{j}, \bar{w}\right)$ to either $(\infty, \infty)$ or $(\infty, m)$, where $m \in(0, \infty)$. But, in any case, the projection of continuum $\Gamma_{j}$ is unbounded on the $b$-axis.

\section{Data Availability}

Data sharing is not applicable to this article as no datasets were generated.

\section{Conflicts of Interest}

All authors declare no conflicts of interest.

\section{Authors' Contributions}

The authors claim that the research was realized in collaboration with the same responsibility. All authors read and approved the last version of the manuscript. 


\section{Acknowledgments}

This work was supported by the National Natural Science Foundation of China (no. 11671322).

\section{References}

[1] I. Prigogine and R. Lefever, "Symmetry breaking instabilities in dissipative systems II," The Journal of Chemical Physics, vol. 48, pp. 1665-1700, 1968.

[2] K. J. Brown and F. A. Davidson, "Global bifurcation in the Brusselator system," Nonlinear Analysis: Theory, Methods \& Applications, vol. 24, no. 12, pp. 1713-1725, 1995.

[3] M. Ghergu, "Non-constant steady-state solutions for Brusselator type systems," Nonlinearity, vol. 21, no. 10, pp. 2331-2345, 2008.

[4] M. Ghergu and V. Rădulescu, "Turing patterns in general reaction-diffusion systems of Brusselator type," Communications in Contemporary Mathematics, vol. 12, no. 4, pp. 661-679, 2010.

[5] G.-h. Guo, J.-h. Wu, and X.-h. Ren, "Hopf bifurcation in general Brusselator system with diffusion," Applied Mathematics and Mechanics, vol. 32, no. 9, pp. 1177-1186, 2011.

[6] B. Li and M.-x. Wang, "Diffusion-driven instability and Hopf bifurcation in Brusselator system," Applied Mathematics and Mechanics, vol. 29, no. 6, pp. 825-832, 2008.

[7] Y. Li, "Hopf bifurcations in general systems of Brusselator type," Nonlinear Analysis: Real World Applications, vol. 28, pp. 32-47, 2016.

[8] M. Liao and Q. Wang, "Stability and bifurcation analysis in a diffusive Brusselator-type system," International Journal of Bifurcation and Chaos in Applied Sciences and Engineering, vol. 26, pp. 1-11, 2016.

[9] M. Ma and J. Hu, "Bifurcation and stability analysis of steady states to a Brusselator model," Applied Mathematics and Computation, vol. 236, pp. 580-592, 2014.

[10] S. Ma, "The stochastic Hopf bifurcation analysis in Brusselator system with random parameter," Applied Mathematics and Computation, vol. 219, no. 1, pp. 306-319, 2012.

[11] R. Peng and M. Wang, "Pattern formation in the Brusselator system," Journal of Mathematical Analysis and Applications, vol. 309, no. 1, pp. 151-166, 2005.

[12] R. Peng and M. Yang, "On steady-state solutions of the Brusselator-type system," Nonlinear Analysis: Theory, Methods \& Applications, vol. 71, no. 3-4, pp. 1389-1394, 2009.

[13] S. H. Strogatz, Nonlinear Dynamics and Chaos with Applications to Physics, Biology, Chemistry, and Engineering, Addison-Wesley, New York, NY, USA, 1994.

[14] R. P. Agarwal and P. J. Y. Wong, Advance Topics in Difference Equations, Kluwer, Dordrecht, Netherlands, 1997.

[15] J. D. Murray, Mathematical Biology, Springer, New York, NY, USA, 1989.

[16] Q. Din, "A novel chaos control strategy for discrete-time Brusselator models," Journal of Mathematical Chemistry, vol. 56, no. 10, pp. 3045-3075, 2018.

[17] H. Kang and Y. Pesin, "Dynamics of a discrete Brusselator model: escape to infinity and Julia set," Milan Journal of Mathematics, vol. 73, no. 1, pp. 1-17, 2005.

[18] W. G. Kelley and A. C. Peterson, Difference Equations: An Introduction with Applications, Harcourt/Academic Press, San Diego, CA, USA, 2nd edition, 2001.

[19] L. Xu, L. J. Zhao, and Z. X. Chang, "Turing instability and pattern for mation in a semi-discrete Brusselator model," Mod. Phys. Lett. B.vol. 27, pp. 1-9, 2013.
[20] H. Kang, "Dynamics of local map of a discrete Brusselator model: eventually trapping regions and strange attractors," Discrete \& Continuous Dynamical Systems-A, vol. 20, no. 4, pp. 939-959, 2008.

[21] R. C. Mittal and R. Jiwari, "Numerical solution of two-dimensional reaction-diffusion Brusselator system," Applied Mathematics and Computation, vol. 217, no. 12, pp. 54045415, 2011.

[22] E. H. Twizell, A. B. Gumel, and Q. Cao, "A second-order scheme for the "Brusselator" reaction-diffusion system," Journal of Mathematical Chemistry, vol. 26, no. 4, pp. 297316, 1999.

[23] R. Ma, C. Gao, and Y. Lu, "Spectrum of discrete second-order Neumann boundary value problems with sign-changing weight," Abstract and Applied Analysis, vol. 2013, Article ID 280508, 10 pages, 2013.

[24] P. H. Rabinowitz, "Some global results for nonlinear eigenvalue problems," Journal of Functional Analysis, vol. 7, no. 3, pp. 487-513, 1971. 